\title{
Lumbar disc degeneration and genetic factors are the main risk factors for low back pain in women: the UK Twin Spine Study
}

\author{
Gregory Livshits, ${ }^{1,2}$ Maria Popham, ${ }^{1}$ Ida Malkin, ${ }^{2}$ Philip N Sambrook, ${ }^{3}$ \\ Alex J MacGregor, ${ }^{1,4}$ Timothy Spector, ${ }^{1}$ Frances M K Williams ${ }^{1}$
}

\begin{abstract}
- A supplementary table is published online only. To view this file please visit the journal online (http://ard.bmj.com).

'Department of Twin Research and Genetic Epidemiology, King's College London, London, UK

2Department of Anatomy and Anthropology, Tel Aviv University, Tel Aviv, Israel ${ }^{3}$ Bone and Joint Research, Kolling Institute, University of Sydney, Sydney, New South Wales, Australia

${ }^{4}$ School of Medicine, University of East Anglia, Norwich, UK
\end{abstract}

\section{Correspondence to} Dr Frances M K Williams, Department of Twin Research and Genetic Epidemiology, King's College London, St Thomas' Hospital, London SE1 7EH, UK; frances.williams@kcl.ac.uk

Accepted 1 May 2011 Published Online First 6 June 2011

\author{
ABSTRACT \\ Objective Low back pain (LBP) is a common \\ musculoskeletal disorder, but it is still unclear which \\ individuals develop it. The authors examined the \\ contribution of genetic factors, lumbar disc degeneration \\ (LDD) and other risk factors in a female sample of the \\ general population.
}

Material and Methods A cross-sectional study was conducted among 2256 women (371 and 698 monozygotic and dizygotic twin pairs and 29 sibling pairs and 60 singletons) with a mean age of 50 years (18-84). A self-reported validated questionnaire was used to collect back pain data. Risk factors including body weight, smoking, occupation, physical exercise and MRI assessed LDD were measured. Data analysis included logistic regression and variance decomposition.

Results The major factors associated with LBP included genetic background, with OR approximately 6 if the monozygotic co-twin had LBP, or 2.2 if she was a dizygotic co-twin. In addition, LDD and overweight were highly significantly $(p<0.001)$ associated with non-specific LBP. The single most important risk factor was the amount of LDD. After adjustment for other risk factors, the individuals who exhibited advanced LDD (90\% vs 10\%) had 3.2 higher odds of manifesting LBP. The data also showed a significant $(p<0.001)$ genetic correlation between the LBP and LDD measurements, suggesting that approximately $11-13 \%$ of the genetic effects are shared by LDD and LBP.

Conclusions The main risk factors for reported episodes of severe and disabling LBP in UK women include the degree of LDD as assessed by MRI, being overweight and genetic heritability.

Low back pain (LBP) is a common musculoskeletal condition in all ages worldwide ${ }^{12}$ and in Europe in particular. ${ }^{3}$ The lifetime prevalence of non-specific LBP may reach $80 \%$, with the annual prevalence ranging between $25 \%$ and $60 \%$ in different ethnic groups. ${ }^{4-6}$ Radiological features of lumbar disc degeneration (LDD) are almost universal in adults, ${ }^{6}$ and have been proposed repeatedly as one of the main causes of LBP. ${ }^{7-9}$ Although an association has been demonstrated, ${ }^{10} 11$ the individual risk factors for LBP remain unclear and universal consensus regarding the extent of LDD-LBP association is lacking. ${ }^{12-15}$ The lack of standardised clinical criteria and radiological definitions has further hampered the undertaking of well-executed epidemiological studies. More sensitive imaging modalities, such as MRI, have fallen in cost and become more widely available and are paving the way for new, largescale epidemiological studies of LDD.

Obesity, smoking and occupation have been reported to be associated with prevalent LBP, although the quantitative effect of the majority of them has been found to be negligible-even insignificant. ${ }^{16-19}$ On the other hand, several studies have consistently suggested the presence of a major genetic component underlying variation of LBP, 1020 although data remain scarce.

The major aim of this study, representing a continuation of our preliminary study, ${ }^{10}$ was to evaluate the association of non-specific LBP with LDD and putative genetic factors in a sample taken from the general population, using extensive data from the UK Twin Spine Study (TUTSS). The UK Twin Spine Study has one of the world's largest samples for whom LBP data and important risk factors, including MRI-determined LDD status, are available in a subsample. Other relevant risk factors available for this study included age, anthropometrics, smoking and physical exercise. This allowed us to conduct rigorous association analysis of the LBP with LDD scores and genetic heritability, simultaneously controlling for the effect of other risk factors. Needless to say, the study does not consider several other potentially relevant risk factors, including inflammatory disease, fracture or the psychological component in back pain, which is an important contributor to pain and disability. ${ }^{21}$

\section{PATIENTS AND METHODS \\ Study sample}

A total of 2256 women (age range 18-84 years, with a mean of 50 years) was examined in this project and included 371 and 698 pairs of monozygotic and dizygotic twin pairs, 29 pairs of siblings and 60 singletons. This sample was taken from the TwinsUK register (http://www.twinsuk.ac.uk), a volunteerbased group of healthy adult twins drawn from the UK population. ${ }^{22}$ Participating twins underwent a nurse-led interview and had been sent, before their visit, a standardised questionnaire for completion, without conferring, relating to lifetime history of low back symptoms. The low back questions followed the format of the Medical Research Council Nurses Study, ${ }^{23}$ and included both questions and a mannequin diagram on which to demonstrate pain distribution. LBP was defined as being located between the 12th ribs and the gluteal folds. Pain associated with fever, menstruation or pregnancy was excluded. Our analyses focused on 
pain associated with disability in 'activities of daily living' (see below), with LBP_12 defined as pain associated with disability that has lasted more than 1 day during the previous 12 months; LBP_1 was defined as pain associated with disability lasting more than 1 month ever. LBP1 (and LBP12) was defined as a binary trait (ie, 1 affected vs 0 non-affected) but we also considered LBP_12Q and LBP_1O as semiquantitative traits, in which the degree of LBP was defined as the number of daily tasks not achievable in LBP episodes during the past 12 months and ever, respectively. 'Activities of daily living' were defined as any one of the following being impossible to achieve (as opposed to just 'difficult'): walking around the house; standing for $15 \mathrm{~min}$; getting up from a low chair; getting out of the bath; getting in and out of the car; going up and down stairs; putting on socks or stockings and cutting toenails. Details of potential confounding variables were recorded at the interview: height; weight; history of smoking; history of manual work including physical occupations such as cleaning or lifting and physical exercise. Physical exercise was defined by a positive answer to 'Have you done any regular sporting activity at any time since you left school?' Ethics committee approval had been obtained and the participants gave informed consent.

LDD status was established using MRI, performed in 909 subjects using a Siemens (Munich, Germany) 1.0-tesla superconducting magnet between 1998 and 2001. The method is described in detail elsewhere. ${ }^{24}$ The following radiographic coding on a scale of $0-3$ was made for each of the five intervertebral discs in the lumbar spine: (1) disc signal intensity within the nucleus pulposus; (2) disc height; (3) disc extension and (4) anterior osteophytes. Each trait code was summed over the five lumbar discs to produce a summary variable (LSIG, LHT, LBUL, LOST) and the summary variables were added to provide an overall summary score, LSUM.

\section{Statistical and genetic analysis}

The analysis was conducted in two stages. First, identification of the potential confounder effect on LBP was examined. We conducted a series of variance (for discrete LBP variables) and correlation (for semiquantitative LBP variables) analyses with LSUM and its structural components with the above confounders as independent predictors. To verify the consistency and the extent of the associations between the four LBP variables and LDD traits we performed canonical correlation analysis between these two arrays of traits. This measures the association of two canonical variables, one representing a set of dependent variables (LBP status variables), the other a set of independent variables (LDD traits, etc) and evaluates simultaneously the contribution of each variable to the observed correlation. Variables showing significant association with LBP status were used to predict the risk of LBP in multiple logistic regression analysis. We evaluated the extent of association of the cumulative degenerative score, LSUM, and its components with LBP1, simultaneously adjusting for age, weight and other predictors for the risk of LBP. This analysis also included the LBP status of the twin, and estimated the OR added by affected dizygotic and monozygotic twin, respectively.

In the second stage, the effects of potential covariates (eg, age, weight) on LBP variation were estimated simultaneously with the effect of the putative genetic factors, using variance component analysis. ${ }^{25}$ In these analyses, the total variance $\left(\mathrm{V}_{\mathrm{PH}}\right)$ of the phenotype of interest (LBP, LSUM) is decomposed into genetic $\left(V_{a d}\right)$ and environmental components. The latter in turn may include common family environment $\left(\mathrm{V}_{\mathrm{CM}}\right)$ and unexplained residual influences $\left(V_{\mathrm{RS}}\right)$. Theoretically, monozygotic twins have all their genes in common, while dizygotic pairs share on average half of their segregating genes. When the phenotypic correlation between monozygotic twin pairs is greater than that of dizygotic twin pairs, it is assumed that genetic influences underlie the increased familial aggregation. Using the maximum likelihood ratio test (LRT) as a model-fitting technique, a best fitting and most parsimonious model was obtained after excluding all non-significant parameters from the general model (backward elimination).

As significant correlations were observed between LBP and LDD variables, we assumed that these correlations were attributable to common genes and/or common environmental factors. To test this hypothesis we used bivariate variance component analysis, which evaluates to what extent the observed phenotypic correlation can be caused by pleiotropic genetic factors (measured by additive genetic correlation, $\mathrm{r}_{\mathrm{G}}$ ), and/or by shared environmental factors (measured by environmental correlation, $r_{E}$ ). ${ }^{26}$ While genetic correlation is a quantitative measure of the shared effects of genes on each of the two traits under analysis, environmental correlation estimates the extent to which these traits share a common environment.

\section{RESULTS}

\section{Descriptive statistics and covariates effect}

The preliminary statistical analysis showed highly significant $(p<0.001)$ correlations between all LBP variables. We therefore selected two moderately intercorrelated $(r=0.62)$ variables, LBP1 and LBP_1O (both defined in the Patients and methods section), for further analysis. These variables were selected because they reflect pain episodes lasting for more than 1 month over life time and therefore are a more reliable measure than LBP_12 variables. The second reason was the results of the canonical and regular correlation analyses showing LBP_1 to have the strongest association with LDD phenotypes (see below). As we are interested in the consistency of the results with respect to other LBP phenotypes, we chose semiquantitative phenotype LBP_1O (vs binary LBP_1). The prevalence of LBP1 in the total sample was approximately $24 \%$ with no difference between the dizygotic and monozygotic twins (0.242 vs 0.243 ). However, the concordance rate in the total sample was significantly higher in monozygotic versus dizygotic twins $\left(\chi_{(2)}^{2}=8.02, \mathrm{p}=0.018\right)$, with corresponding tetrachoric correlations, 0.641 and 0.375 , respectively ( $\mathrm{p}<0.001$, for both).

Table 1 shows basic characteristics of the twins and the available sample sizes by LBP1 status. Women with disabling episodes of back pain were more likely to be older, heavier and taller, to have done manual work and to perform physical exercise. In addition, all LDD and LBP variables were significantly higher in the LBP1 group. We found no association between LBP1 and smoking, and age had a minimal effect on back pain status. As weight and body mass index (BMI) were strongly correlated $(\mathrm{r}=0.91, \mathrm{p}<0.001)$, to avoid redundancy and collinearity in multivariable analysis, and as weight was more highly correlated with LBP than BMI, only weight was considered in the following analysis.

As associations between all LDD traits and LBP status were highly statistically significant (table 1), we tested the consistency of the associations using correlation and canonical correlation analyses (table 2). The latter was performed on adjusted and non-adjusted variables and showed a highly significant $(p<0.001)$ association between the two arrays of variables. The magnitude of canonical correlations was approximately the 
Table 1 Characteristics of the UK Twins Spine study sample by LBP status

\begin{tabular}{|c|c|c|c|c|c|c|c|}
\hline \multirow{2}{*}{$\begin{array}{l}\text { Predictor } \\
\text { variable }\end{array}$} & \multicolumn{3}{|c|}{ LBP1 absent } & \multicolumn{3}{|c|}{ LBP1 present } & \multirow{2}{*}{$\begin{array}{l}\text { ANOVA }(F) \text {, } \\
\text { p value }\end{array}$} \\
\hline & $x$ & SD & $\mathbf{N}$ & $x$ & SD & $\mathbf{N}$ & \\
\hline Age (year) & 49.95 & 11.78 & 1710 & 51.48 & 10.28 & 546 & $7.34,0.007^{*}$ \\
\hline Height (m) & 1.62 & 0.06 & 1710 & 1.63 & 0.06 & 546 & $12.00,0.0004^{*}$ \\
\hline Weight (kg) & 64.89 & 11.32 & 1709 & 68.97 & 12.93 & 544 & $49.97,0.0001 *$ \\
\hline BMI $\left(\mathrm{kg} / \mathrm{m}^{2}\right)$ & 24.69 & 4.29 & 1709 & 25.92 & 4.79 & 544 & $33.21,0.0001^{*}$ \\
\hline $\begin{array}{l}\text { Smoking (packs/ } \\
\text { year) }\end{array}$ & $\begin{array}{c}4.10 \\
(672.70)^{\dagger}\end{array}$ & 9.68 & 1050 & $\begin{array}{l}4.19 \\
(689.72)^{\dagger}\end{array}$ & 10.27 & 302 & $0.7,0.413^{\dagger}$ \\
\hline Manual work & $\begin{array}{c}2.19 \\
(1120.03)^{\dagger}\end{array}$ & 6.46 & 1710 & $\begin{array}{l}2.97 \\
(1208.80)^{\dagger}\end{array}$ & 6.92 & 546 & $5.80,0.016^{\dagger}$ \\
\hline PhysExers ${ }^{\ddagger}$ (yes) & $60.73 \%$ & & 753 & $80.76 \%$ & & 361 & $\chi^{2}=58.8,<0.0001$ \\
\hline LHT & 3.45 & 2.56 & 654 & 4.93 & 2.83 & 182 & $45.40,0.001^{*}$ \\
\hline LSIG & 5.70 & 3.28 & 656 & 7.07 & 2.99 & 183 & $25.96,0.001^{*}$ \\
\hline LBUL & 2.00 & 1.74 & 656 & 2.86 & 1.84 & 183 & $33.87,0.001^{*}$ \\
\hline LOST & 1.35 & 1.85 & 654 & 1.99 & 2.42 & 183 & $14.98,0.001^{*}$ \\
\hline LSUM & 12.52 & 7.30 & 652 & 16.82 & 7.92 & 182 & $47.67,0.001^{*}$ \\
\hline LBP1_Q" & $\begin{array}{c}0.714 \\
(902.75)^{\dagger}\end{array}$ & 1.887 & 1730 & $\begin{array}{l}5.083 \\
(1889.75)^{\dagger}\end{array}$ & 2.622 & 522 & $1256.02,0.0001^{\dagger}$ \\
\hline
\end{tabular}

LBP 1 represents low back pain (LBP) defined as pain associated with disability lasting more than a month over life time (other LBP phenotypes defined in the Patients and methods section).

*The analysis of variance (ANOVA) result was confirmed by Kruskal-Wallis test.

${ }^{\dagger}$ Kruskal-Wallis test $\left(\chi^{2}\right.$ and $p$ value), mean group ranks are provided in parentheses.

‡Percentage who exercised.

§Percentage who complained.

"LBP1-absent individuals can have LBP1 $0>0$, if the length of time they have had back pain is less than 1 month throughout

their whole life. For an individual to be LBP1-present, they need to have disabling pain lasting longer than 1 month.

BMI, body mass index; LBUL, disc bulge; LHT, disc height; LOST, anterior osteophytes; LSIG, disc signal intensity;

LSUM, summary rank for four lumbar disc degeneration traits; N, sample size; $X$, mean or proportion.

same $(R \sim 0.250)$ regardless of adjustment and whether LSUM or its four structural components were used. Considering LBP and LDD variables adjusted for significant covariates (table 2), it is worth mentioning that canonical weights (not shown), representing a variable's unique contribution, were of comparable magnitude for LSUM components, ranging from 0.278 (LSIG) to 0.409 (LHT). However, the contribution of LBP variables varied much more, from 0.179 (LBP_1Q) to 0.853 (LBP1). One interpretation of these findings is that all four MRI traits make a significant and similar contribution to LBP, but especially to LBP1.

\section{Independent risk factors for LBP}

This was tested using multiple logistic regression for the effect of all significant variables (table 1) on LBP1. First, to preserve sample size, LDD traits were not included in the analysis. Body weight, exercise and LBP status of the co-twin were highly significantly $(p<0.001$ for each covariate) associated with LBP1. Next, we added the LDD status avoiding collinearity, by examining LSUM and its constituent traits separately. LSUM, weight, physical exercise and LBP1 status of the co-twin were all found to be significant predictors of back pain (table 3). Testing the association with the four MRI traits of LDD, only disc height ( $p=0.0013$ ) and disc bulge $(p=0.0311)$ were independent significant predictors. However, the OR of each of them was substantially lower than the corresponding effect for the summary trait LSUM. Comparison of the upper versus the lower $10 \%$ of the predictor variables distribution suggested that LSUM is the major potential risk factor for LBP (table 3). The contribution of monozygotic co-twins to LBP risk was virtually twice the effect of dizygotic twins: 1.757 (SE 0.317) versus 0.782 (SE 0.253), with $p<0.001$, clearly highlighting the contribution of genetic factors to LBP.

\section{Contribution of genetic factors}

Next we examined the contribution of genetic factors to LBP1. Taking the LBP status of the co-twin into account, logistic regression analysis suggested a significant genetic background
Table 2 Correlation and canonical correlation analysis of LBP and LDD traits

\begin{tabular}{lllll}
\hline LDD variable & \multicolumn{1}{l}{ LBP12 } & LBP1 & LBP_120 & LBP_10 \\
\hline \multicolumn{2}{l}{ Pair-wise correlations } \\
LSUM & 0.13 & 0.25 & 0.16 & 0.22 \\
LHT & 0.10 & 0.23 & 0.09 & 0.18 \\
LBUL & $0.07^{*}$ & 0.19 & 0.10 & 0.16 \\
LOST & 0.16 & 0.12 & 0.20 & 0.18 \\
LSIG & $0.06^{*}$ & 0.18 & 0.08 & 0.14 \\
Canonical & Non-adjusted & LDD-adjusted & LBP-adjusted & Both adjusted \\
correlation, $\mathrm{R}^{\dagger}$ & $0.253 / / 0.261$ & $0.280 / / 0.282$ & $0.228 / / 0.239$ & $0.270 / / 0.272$
\end{tabular}

Pair-wise correlations between the lumbar disc degeneration (LDD) traits and low back pain (LBP) variables adjusted for age, weight and exercise. The definitions for all LDD and LBP variables are given in the Material and Methods section.

* Statistically significant $p<0.005$.

${ }^{\dagger}$ Canonical correlations (R) are given separately for LSUM with all LBP variables, left number and for four LDD components and four LBP variables (right number). All $p$ values for canonical correlations were $<10^{-4}$.

LBUL, disc bulge; LHT, disc height; LOST, anterior osteophytes; LSIG, disc signal intensity; LSUM, summary rank for four LDD traits.

(table 3), which is also supported by high and zygosity-dependent tetrachoric correlations between the twins (see above). The correlations between dizygotic and monozygotic twins in LBP_1O scores were also in accord, 0.374 and 0.467 ( $p<0.001$ for both). We therefore submitted both LBP variables to univariate and bivariate variance component analyses with LSUM (table 4) to evaluate the extent of the genetic influence and to examine the shared genetic effects between LBP and MRIdetermined LDD. We found significant heritability for both LBP variables and LSUM. By LRT, the most parsimonious model (balancing fit of data with minimum complexity) displayed a significant heritability estimate of $43.5 \%$ (LBP1) to $68.2 \%$ (LSUM) of the residual variation adjusted for significant covariates. Both LBP1 and LBP1Q variables displayed highly significant $(p<0.001)$ genetic correlations with LSUM (table 4). However, we found no common environmental factors simultaneously affecting LBP1 (or LBP_1O) and LSUM variations. 
Table 3 Multiple logistic regression of LBP taking into account LDD status effect

\begin{tabular}{|c|c|c|c|c|c|c|}
\hline \multirow[b]{2}{*}{ Risk factor* } & \multicolumn{2}{|c|}{ Coefficient } & \multicolumn{2}{|c|}{ Significance } & \multicolumn{2}{|l|}{ OR (95\% Cl) } \\
\hline & Estimate & Standard error & Wald's $\chi^{2}$ & p Value & For unit & For $90 \%$ vs $10 \%^{\dagger}$ \\
\hline Constant & -4.164 & 0.584 & 50.903 & $<0.0001$ & - & - \\
\hline \multicolumn{7}{|c|}{ Regression coefficients } \\
\hline Weight & 0.021 & 0.008 & 6.773 & 0.0091 & 1.021 (1.005 to 1.037$)$ & 1.869 (1.162 to 2.985$)$ \\
\hline Exercise & 0.481 & 0.208 & 5.319 & 0.0210 & $1.617(1.074$ to 2.435$)$ & $1.617(1.074$ to 2.435$)$ \\
\hline LBP(DZ_sib) & 0.782 & 0.253 & 9.529 & 0.0020 & 2.186 (1.329 to 3.595$)$ & $2.186(1.329$ to 3.595$)$ \\
\hline
\end{tabular}

*All variables showing significant association with LBP 1 in the preliminary analysis (table 1) were included into a multiple logistic regression analysis. However, only variables making independent significant contributions are presented in the table. To account for contribution of the affection status of siblings in the logistic regression model, including twins of different zygosity, the following definitions were introduced: $L B P(D Z$ sib $)=1$ if an individual has affected dizygotic sibling, otherwise 0 ; LBP(MZ sib)=1 if an individual has affected monozygotic sibling, otherwise 0 .

${ }^{\dagger}{ }^{\prime} 90 \%$ vs $10 \%$ ' means that OR was estimated contrasting the variable upper vs lower 10 percentiles of the distribution. The corresponding results of univariate analysis of the LSUM, weight and affected twin zygosity effect on individual probability to have LBP1 are shown in the supplementary table. There were 49\% LBP1 individuals in the fourth quartile of LSUM distribution, compared with only $6.9 \%$ in the first one. Comparison of the first affected monozygotic vs dizygotic twin effect on the probability that the second twin will have LBP1, were statistically reliably significant $\left(\chi^{2}=10.99, \mathrm{df}=1, \mathrm{p}=0.0009\right)$.

DZ, dizygotic; LBP, low back pain; LDD, lumbar disc degeneration; LSUM, summary rank for four LDD traits; MZ, monozygotic.

Table 4 Sharing of genetic and environmental parameters between LDD and LBP: bivariate variance component analysis

\begin{tabular}{llll}
\hline Parameter & LBP1 $^{*}$ & LSUM & LBP_10 \\
\hline$\sigma_{\mathrm{AD}}{ }^{2}$ & $0.399 \pm 0.049$, & $28.315 \pm 2.665$, & $3.608 \pm 0.288$, \\
$\sigma_{\mathrm{RS}}{ }^{2}$ & $\mathrm{~h}^{2}=0.435^{\dagger}$ & $\mathrm{h}^{2}=0.682^{\dagger}$ & $\mathrm{h}^{\dagger}=0.566^{\dagger}$ \\
$\alpha_{0}$ & $0.519 \pm 0.042$ & $13.238 \pm 1.515$ & $2.763 \pm 0.202$ \\
$\beta_{\text {EXCERCISE }}$ & $-0.0763 \pm 0.0282$ & $-18.056 \pm 2.224$ & $-0.8557 \pm 0.3754$ \\
$\beta_{\text {WEIGHT }}$ & - & $\mathrm{NS}$ & $0.6919 \pm 0.1337$ \\
$\beta_{\text {AGE }}$ & - & $0.0859 \pm 0.0203$ & $0.0276 \pm 0.0054$ \\
$\mathrm{R}_{\text {AD }}$ & $0.3662 \pm 0.0842$ & $0.4831 \pm 0.0326$ & - \\
\multicolumn{1}{c}{$\mathrm{R}_{\mathrm{RS}}$} & $(\mathrm{p}<0.001)$ & & $0.3318 \pm 0.0705$ \\
& $0.0910 \pm 0.080$ & & $(\mathrm{p}<0.001)$ \\
& $(\mathrm{p}=0.29)$ & & $0.0815 \pm 0.0786$ \\
\hline
\end{tabular}

* LBP1 variable was adjusted using parameter estimates in multiple logistic regression (table 3), in which the contribution of the low back pain (LBP) status of twins was not included; Parameter estimates \pm standard errors of estimates are provided in the table. ${ }^{\dagger}$ Contribution of the putative genetic effects (heritability estimate), adjusted for all significant covariates; $\sigma_{\mathrm{AD}}{ }^{2}, \sigma_{\mathrm{RS}}{ }^{2}$ are variance components attributable to genetic and random environmental effects on each variable separately; $\alpha_{0}$ and $\beta$ are regression parameters, intercept and slope for the corresponding significant covariates; $R_{A D}$ and $\mathrm{R}_{\mathrm{BS}}$ are genetic and environmental correlations between LSUM and each of the two LBP variables respectively; $\mathrm{p}$ value in comparison with the corresponding general model in which all model parameters were estimated.

LDD, lumbar disc degeneration; LSUM, summary rank for four LDD traits.

\section{DISCUSSION}

The major aim of this study was to assess to what extent genetic factors and LDD contribute to reports of previous episodes of non-specific LBP within the middle-aged female population. As consistency is lacking in the association between back pain and the degeneration process of intervertebral discs, ${ }^{11} 1527$ the contribution of common genetic and non-genetic factors with their potential association are of interest. The main results of this study, summarised in figure 1, show a major contribution of the specific genetic factors to variation of the LBP1 and LDD phenotypes, respectively. This figure illustrates a potentially strong effect of LDD on LBP1 appearance, and also the extent of association of both LBP1 and LDD with common covariates. The major risk factors for LBP were genetic factors, which gave OR of approximately 6 , if the monozygotic co-twin had LBP or 2.2 (if she was a dizygotic co-twin), being overweight and, the most important, the amount of MRI-determined LDD. Therefore, after adjustment for other risk factors, the individuals who exhibited advanced LDD (90\% vs $10 \%$ ) had 3.2 higher odds of manifesting LBP. The data also showed the existence of a significant genetic correlation between LBP and
0.12

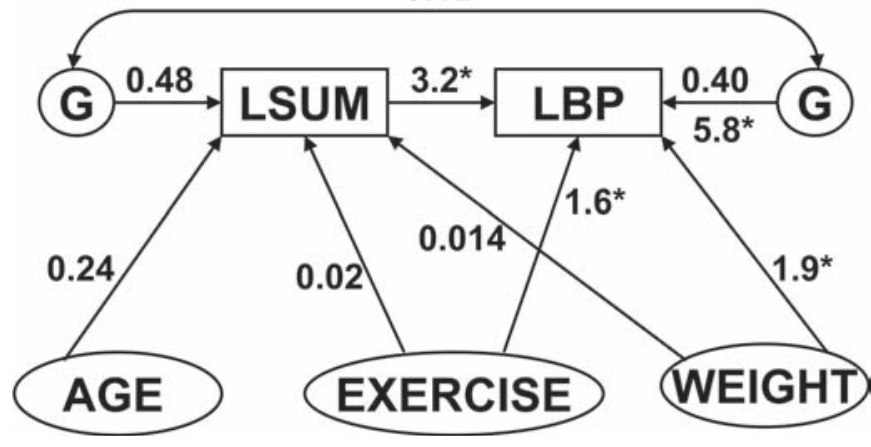

Figure 1 Path diagram of the main risk factors for low back pain (LBP) in women. The figures show proportions of the total variance attributable to an independent effect of the corresponding predictor variables and $\mathrm{OR}$ (marked by ${ }^{*}$ ). The $\mathrm{OR}$ are from table 3 . The heritability estimates as presented in the figure are unadjusted to show their relative effect in comparison with other risk factors, and were obtained at the preliminary stage before final bivariate analysis presented in table 4. The variance components for lumbar disc degeneration, including unadjusted estimates of heritability, were also obtained in the corresponding univariate variance component analysis, used next in the bivariate analysis with LBP.

LSUM measurements. The $\mathrm{R}^{2}{ }_{\mathrm{AD}}$ suggests ${ }^{28}$ that approximately $11-13 \%$ of the genetic effects were shared in this sample. Similar results were obtained when we tested binary trait LBP1, and with the semicontinuous trait LBP_1O. The parameter estimates in these analyses (table 4) are probably not very accurate, but they are nevertheless highly significant by LRT. The substantial genetic component in LBP1 observed in variance component analysis is confirmed by concordance rates differing between the monozygotic and dizygotic twins (tetrachoric correlation in monozygotic vs dizygotic twins 0.641 vs 0.375 , $\mathrm{p}=0.018$ ) and by multiple logistic regression analysis (table 3 ). In the total sample, the regression coefficient $\beta$ for the dizygotic twin effect was $0.782 \pm 0.253$ (with OR 2.19); however, if the sibling is monozygotic the effect was virtually twice as large (1.757 \pm 0.317 , OR 5.79), and reliably significant, $p=0.0009$.

There was notable agreement between the results of multiple logistic regression of the risk of LBP and bivariate genetic analysis (tables 3 and 4). The former suggests that the disc degeneration is a highly important risk factor for LBP $(\mathrm{p}<0.0001)$ and remains such in univariate and multivariate analyses, regardless 
of adjustment. While several studies have been equivocal in reporting an association between LDD and LBP, ${ }^{15-17}$ other studies of large samples find a convincing correlation between LBP and the severity of MRI LDD. 8911 In our study, age was a major risk factor for LDD, but of interest, it exerted no independent effect on the risk of reported episodes of LBP.

The present results are in good agreement with published limited data, including a previous study from TwinsUK ${ }^{10}$ suggesting a significant contribution of genetic factors to the variation of different indicators of LBP in adults. ${ }^{20}$ The latter study used several back pain definitions in male twins, and found substantially higher intraclass correlations for all of them in monozygotic twins (compared with dizygotic). These led to heritability estimates of $0.30-0.46$, which are comparable to estimates obtained in the present study, but at odds with the findings in Finnish children ${ }^{29}$ that twins' shared environment, and not genetic factors, is responsible for the similarity in LBP status. Contrary to this, and in agreement with other studies using adults, ${ }^{20} 30$ including a preliminary study of this sample, ${ }^{10}$ our estimates gave significantly higher tetrachoric correlations in monozygotic versus dizygotic twins (0.641 vs $0.375, p<0.02$ for difference) and substantial estimates of heritability.

Our data are also in agreement with the Battie study ${ }^{20}$ with respect to a significant estimate of heritability for LHT and LBP, and genetic correlation between the two, despite the different assessment of LBP in the two studies. The studies agree that only a portion of the genetic effects on LBP was caused by genes governing the variation of MRI variables, and an even smaller portion by environmental factors shared by LBP and LSUM. This suggests the existence of specific genetic effects and additional risk factors for both LBP and LSUM. Of these, we highlight weight, which in our study showed that overweight individuals have a greater risk of LBP in comparison with lean women (table 3). Significant genetic association between LSUM and LBP may have an important implication for future studies. It may suggest the existence of common metabolic pathways leading to the manifestation of both phenotypes and thus direct research towards the specific candidate genes, such as those involved in the metabolism and ageing of nucleus pulposus. LBP and LDD is governed by some common, but mostly independent, genetic factors the nature of which remain to be determined. 31

Certain limitations can be recognised in this study. Based on a female sample the results should not be extrapolated to men-there is also less exposure to heavy manual work in our subjects. Although we are not aware of the sex-specific genetic effects for LBP, ${ }^{32}$ differences may exist in the physiological background, as well as in lifestyle. The second limitation is the cross-sectional design of the study to derive conclusions in an age-dependent condition. Finally, questionnaire assessment of pain is subjective and may not be fully comparable between studies. In the present project, however, there was a very strong association between different LBP measures, as seen in table 1 , and a remarkable similarity in association between them and LDD variables in canonical correlation analysis (table 2 ).

In conclusion, this cross-sectional study of a large sample of female twins shows that in addition to genetic predisposition, episodes of LBP are strongly associated with the extent of LDD as assessed by MRI. In addition, genetic variation of LBP and LDD is governed by some common but mostly independent genetic factors, the nature of which remain to be determined.
Acknowledgements The authors would like to thank all the twins who participated in the study, staff in the Department of Twin Research and to acknowledge financial support from the Wellcome Trust, the Department of Health via the National Institute for Health Research (NIHR) comprehensive Biomedical Research Centre award to Guy's \& St Thomas' NHS Foundation Trust in partnership with King's College London and the Arthritis Research Campaign. The study was also partly supported by the Israeli Ministry of Health, chief scientist grant no 3-4101.

Funding The Wellcome Trust, the Arthritis Research Campaign, Israeli Ministry of Health chief scientist.

Competing interests None.

Patient consent Obtained.

Ethics approval This study was conducted with the approval of the St Thomas Research Ethics Committee.

Provenance and peer review Not commissioned; externally peer reviewed.

\section{REFERENCES}

1. Maniadakis N, Gray A. The economic burden of back pain in the UK. Pain 2000;84:95-103.

2. Brooks PM. The burden of musculoskeletal disease - a global perspective. Clin Rheumatol 2006;25:778-81.

3. European Commission. Special Eurobarometer. European Commission, 2007:272e.

4. McBeth J, Jones K. Epidemiology of chronic musculoskeletal pain. Best Pract Res Clin Rheumatol 2007;21:403-25.

5. Louw QA, Morris LD, Grimmer-Somers K. The prevalence of low back pain in Africa: a systematic review. BMC Musculoskelet Disord 2007;8:105.

6. Andersson GB. Epidemiological features of chronic low-back pain. Lancet 1999;354:581-5

7. Kjaer $\mathbf{P}$, Leboeuf-Yde C, Korsholm L, et al. Magnetic resonance imaging and low back pain in adults: a diagnostic imaging study of 40-year-old men and women. Spine 2005;30:1173-80.

8. Natarajan RN, Williams JR, Lavender SA, et al. Relationship between disc injury and manual lifting: a poroelastic finite element model study. Proc Inst Mech Eng $\mathrm{H}$ 2008;222:195-207.

9. Cheung KM, Karppinen J, Chan D, et al. Prevalence and pattern of lumbar magnetic resonance imaging changes in a population study of one thousand forty-three individuals. Spine (Phila Pa 1976) 2009;34:934-40.

10. MacGregor AJ, Andrew T, Sambrook PN, et al. Structural, psychological, and genetic influences on low back and neck pain: a study of adult female twins. Arthritis Rheum 2004:51:160-7.

11. de Schepper El, Damen J, van Meurs JB, et al. The association between lumbar disc degeneration and low back pain: the influence of age, gender, and individual radiographic features. Spine 2010;35:531-6.

12. Jensen MC, Brant-Zawadzki MN, Obuchowski N, et al. Magnetic resonance imaging of the lumbar spine in people without back pain. N Engl J Med 1994;331:69-73

13. Jarvik JJ, Hollingworth W, Heagerty P, et al. The Longitudinal Assessment of Imaging and Disability of the Back (LAIDBack) Study: baseline data. Spine 2001;26:1158-66.

14. Carragee EJ, Alamin TF, Miller JL, et al. Discographic, MRI and psychosocial determinants of low back pain disability and remission: a prospective study in subjects with benign persistent back pain. Spine J 2005;5:24-35

15. Kalichman L, Kim DH, Li L, et al. Computed tomography-evaluated features of spinal degeneration: prevalence, intercorrelation, and association with self-reported low back pain. Spine J 2010;10:200-8.

16. Battié MC, Videman T. Lumbar disc degeneration: epidemiology and genetics, J Bone Joint Surg Am 2006;88(Suppl 2):3-9.

17. Macfarlane GJ, Pye SR, Finn JD, et al. Investigating the determinants of international differences in the prevalence of chronic widespread pain: evidence from the European Male Ageing Study. Ann Rheum Dis 2009;68:690-5.

18. Shiri R, Karppinen J, Leino-Arjas P, et al. The association between smoking and low back pain: a meta-analysis. Am J Med 2010;123:87.e7-35.

19. Shiri R, Karppinen J, Leino-Arjas P, et al. The association between obesity and low back pain: a meta-analysis. Am J Epidemiol 2010;171:135-54.

20. Battié MC, Videman T, Levalahti E, et al. Heritability of low back pain and the role of disc degeneration. Pain 2007;131:272-80.

21. Deyo RA, Weinstein JN. Low back pain. N Engl J Med 2001;344:363-70.

22. Spector TD, Williams FM. The UK Adult Twin Registry (TwinsUK). Twin Res Hum Genet 2006;9:899-906.

23. Smedley J, Inskip H, Cooper C, et al. Natural history of low back pain. A longitudina study in nurses. Spine 1998;23:2422-6.

24. Sambrook PN, MacGregor AJ, Spector TD. Genetic influences on cervical and lumbar disc degeneration: a magnetic resonance imaging study in twins. Arthritis Rheum 1999:42:366-72 
25. Malkin I, Ginsburg E. Program Package for Pedigree Analysis (Version MAN-2009): Technical Report. Tel Aviv: Department of Anatomy and Anthropology, Tel Aviv University, 2009

26. Falconer DS, Mackay TFC. Introduction to Quantitative Genetics. 4th edn. Harlow: Longmans Green 1996:122-324.

27. Pransky G, Buchbinder R, Hayden J. Contemporary low back pain research - and implications for practice. Best Pract Res Clin Rheumatol 2010;24:291-8.

28. Deng FY, Lei SF, Li MX, et al. Genetic determination and correlation of body mass index and bone mineral density at the spine and hip in Chinese Han ethnicity. Osteoporos Int 2006;17:119-24.
29. El-Metwally A, Mikkelsson M, Ståhl M, et al. Genetic and environmental influences on non-specific low back pain in children: a twin study. Eur Spine J 2008;17:502-8.

30. Hestbaek $\mathbf{L}$, lachine IA, Leboeuf-Yde $\mathrm{C}$, et al. Heredity of low back pain in a young population: a classical twin study. Twin Res 2004; 7:16-26.

31. Liu J, Pei Y, Papasian CJ, et al. Bivariate association analyses for the mixture of continuous and binary traits with the use of extended generalized estimating equations. Genet Epidemiol 2009;33:217-27.

32. Tegeder I, Lötsch J. Current evidence for a modulation of low back pain by human genetic variants. J Cell Mol Med 2009;13:1605-19. 